The Astrophysical Journal SuPPlement SerIES, 105:343-352, 1996 August

(C) 1996. The American Astronomical Society. All rights reserved. Printed in U.S.A.

\title{
SURVEY FOR EMISSION-LINE GALAXIES: UNIVERSIDAD COMPLUTENSE DE MADRID LIST $2^{1}$
}

\author{
J. Zamorano, J. Gallego, M. Rego, A. G. Vitores, ${ }^{2}$ and O. Alonso \\ Departamento de Astrofísica, Universidad Complutense, 28040 Madrid, Spain \\ Received 1995 July; accepted 1995 November 30
}

\begin{abstract}
A low-dispersion objective-prism survey for low-redshift emission-line galaxies (ELGs) is being carried out by the Universidad Complutense de Madrid with the Schmidt telescope at the German-Spanish Observatory of Calar Alto (Almería, Spain). A $4^{\circ}$ full-aperture prism, which provides a dispersion of $1950 \AA \mathrm{mm}^{-1}$, and IIIa-F emulsion combination has been used to search for ELGs selected by the presence of $\mathrm{H} \alpha$ emission in their spectra. A compilation of descriptions and positions, along with finding charts, is presented for 103 emission-line objects. This is the second list, which contains objects located in a region of the sky covering $201.4 \mathrm{deg}^{2}$ in seven fields near $\alpha=15^{\mathrm{h}}$ and $\delta=25^{\circ}$.

Subject headings: galaxies: Seyfert - surveys
\end{abstract}

\section{INTRODUCTION}

The Universidad Complutense de Madrid (UCM) Survey for emission-line objects, which is being carried out at the German-Spanish Observatory of Calar Alto (Spain), has been described in detail by Zamorano et al. (1994, hereafter Paper I, UCM List 1).

The UCM Survey has been initiated with these general objectives: (1) to identify and study new young, lowmetallicity galaxies; (2) to carry out the classification and determination of the overall properties and completeness of the sample of emission-line galaxies (ELGs) selected; (3) to determine the spatial distribution and luminosity function of the new galaxy population; (4) to compare our survey with others and to find out differences between the samples obtained with various objective-prism techniques; (5) to study the overall relation between the far-infrared properties and the optical behavior of the star-forming galaxies; (6) to determine the evolutionary status and the different stellar subjacent populations of the objects in order to detect any effect of evolution in the starburst phenomena; and (7) to quantify the properties of the star formation in the local universe.

The Schmidt telescope of the Calar Alto German-Spanish Observatory (Almería, Spain), equipped with a full-aperture objective prism, is being used in this survey. The $80 / 120 \mathrm{~cm}$ $\mathrm{f} / 3$ Schmidt telescope and $4^{\circ}$ objective prism combination provides a dispersion of $1950 \AA \mathrm{mm}^{-1}$ at $\mathrm{H} \alpha$, and the plate scale is $86^{\prime \prime} \mathrm{mm}^{-1}$ (Birkle 1984). By using IIIa-F emulsion, whose sensitivity decays abruptly at $6850 \AA$, and a RG630 filter, a useful spectral range from 6400 to $6850 \AA$ is selected. Since this bandpass excludes the strong night-sky emission in the blue-visual region, longer exposures can be made without severe plate fogging. This instrumental setup is able to record the $\mathrm{H} \alpha$ emission line for objects up to $z \lessgtr 0.04$.

\footnotetext{
${ }^{1}$ Based on observations collected at the German-Spanish Astronomical Center, Calar Alto, Spain, operated jointly by the Max-Planck-Institut für Astronomie (MPIA), Heidelberg, and the Spanish National Commission for Astronomy. Partly based on observations made with the Isaac Newton Telescope operated on the island of La Palma by the Royal Greenwich Observatory in the Spanish Observatorio del Roque de los Muchachos of the Instituto de Astrofísico de Canarias.

${ }^{2}$ EUIT Industrial Universidad Politécnica, 28012 Madrid, Spain.
}

The IIIa-F plates were hypersensitized by baking them in an atmosphere of $\mathrm{N}_{2}$ gas at $65^{\circ} \mathrm{C}$ for $6 \mathrm{hr}$ prior to exposure.

By careful manual guiding using stars brighter than $m_{v}=$ 9 , and without widening for recording very faint objects, the spectra are generally kept to a width of $0.06 \mathrm{~mm}$. Dispersion runs along the north-south axis. A well-exposed spectrum is about $0.4 \mathrm{~mm}$ long. Field coverage is $5.5 \times 5.5 \mathrm{deg}^{2}$ in plates $24 \mathrm{~cm}$ wide. Plates taken with bad seeing or poor guiding were rejected after visual inspection. Quality standards for the plates analyzed were as follows: (1) good seeing (no worse than $3^{\prime \prime}$ ) and excellent guiding, (2) no cloud interference, (3) no exposure interruption, and (4) telescope near the meridian, i.e., hour angle between $21^{\mathrm{h}} 30^{\mathrm{m}}$ and $2^{\mathrm{h}} 30^{\mathrm{m}}$.

As in Paper I of this series, we present here the list obtained from the second campaign. Follow-up observations, both spectroscopy and imaging, of the UCM galaxies included in the first two lists have been performed in order to address some of the purposes of the survey. Some results have already been published (Gallego et al. 1994; Rego, Zamorano, \& González-Riestra 1989; Rego et al. 1993, 1994; Zamorano et al. 1990, 1992), while others will be published elsewhere (Vitores et al. 1996; Gallego et al. 1996; Alonso-Herrero et al. 1996).

\section{RESULTS}

The plate field centers, dates of observation, seeing, and number of candidates found are listed in Table 1. The total number of galaxies in Zwicky et al. (1961-1968, Catalog of Galaxies and of Clusters of Galaxies, hereafter CGCG) is also tabulated for each plate. These data, obtained from the Catalogue of Principal Galaxies (Paturel et al. 1989, hereafter PGC), are useful to stress the differences between galaxy populations from field to field. A diagram with the fields surveyed is depicted in Figure 1. These plates were taken during an observing run in 1987 June with dark nights and very transparent atmosphere. Although the overlapping spectra of close objects lying together along a north-south axis could lead to spurious identifications, only a small fraction of cases are found in each field that can be eliminated after inspection of direct images on the Palomar Observatory Sky Survey (POSS) prints in order to identify their nature. There is one object that was left out of UCM 
TABLE 1

Journal OF OBSERVATIONS

\begin{tabular}{|c|c|c|c|c|c|c|c|c|}
\hline $\begin{array}{l}\text { Plate } \\
\text { No. }\end{array}$ & $\begin{array}{r}\text { Plate } \\
\text { RA(1950) }\end{array}$ & $\begin{array}{l}\text { Center } \\
\operatorname{DEC}(1950)\end{array}$ & $\begin{array}{c}\text { Date of } \\
\text { Observation }\end{array}$ & UT & $\begin{array}{l}\text { texp } \\
(\min )\end{array}$ & $\begin{array}{l}\text { Seeing } \\
\text { (arcsec) }\end{array}$ & $\begin{array}{l}\text { CGCGs } \\
(z \leq 0.04)\end{array}$ & ELGs \\
\hline A228 & $12^{\text {h }} 58^{m} 3$ & $+29^{\circ} 05^{\prime} 5$ & 1987 jun 20 & $21^{\mathrm{h}} 52^{\mathrm{m}}$ & 120 & 1 & 246 & 46 \\
\hline A229 & $16^{\text {h }} 58^{m} 4$ & $+28^{\circ} 47 ! 9$ & 1987 jun 21 & $01^{h} 00^{m}$ & 180 & 2 & 29 & 11 \\
\hline $\mathbf{A 2 3 0}$ & $16^{\mathrm{h}} 05^{\mathrm{m}} 8$ & $+14^{\circ} 58.7$ & 1987 jun 21 & $22^{\mathrm{h}} 37^{\mathrm{m}}$ & 150 & 2 & 79 & 4 \\
\hline A231 & $16^{\mathrm{h}} 39^{\mathrm{m}} 4$ & $+29^{\circ} 29.5$ & 1987 jun 22 & $01^{\mathrm{h}} 25^{\mathrm{m}}$ & 150 & 2 & 7 & 6 \\
\hline A232 & $13^{\mathrm{h}} 19^{\mathrm{m} 9}$ & $+28^{\circ} 21 ! 2$ & 1987 jun 22 & $21^{\mathrm{h}} 30^{\mathrm{m}}$ & 150 & 3 & 47 & 12 \\
\hline A234 & $14^{h} 41^{m} 4$ & $+27^{\circ} 10^{\prime} 7$ & 1987 jun 23 & $22^{\mathrm{h}} 47^{\mathrm{m}}$ & 150 & 3 & 33 & 24 \\
\hline A236 & $16^{\mathrm{h}} 05^{\mathrm{m}} 0$ & $+21^{\circ} 44^{\prime} .9$ & 1987 jun 24 & $22^{\mathrm{h}} 26^{\mathrm{m}}$ & 129 & 3 & 17 & 2 \\
\hline
\end{tabular}

List 1 (UCM $0023+1908$ ) because it appears as a pair of stellar objects in the north-south direction. Follow-up spectroscopic observations have confirmed the ELG nature of one of the components.

Plates have been carefully searched by visually scanning with a low-power $(10 \times)$ binocular microscope in a scanning frame that allowed coverage in a series of horizontal strips 1 $\mathrm{cm}$ wide. Each plate was inspected by at least three independent observers. A last review of all objects found provides the final selection, which is checked against the POSS plates. The visual inspection of the plates, although efficient, is rather subjective. A study on the feasibility of automatic detection of ELGs using MAMA (Machine Automatique à Mesurer pour l'Astronomie; Guibert \& Moreau 1991) has been started with very promising results (Alonso et al 1995). The automatic procedure will help us to perform objective quality control of the plates and to test the biases of this eye-selected sample.

The candidates were classified according to three parameters. First, the eye estimate of the continuum intensity relative to the plate background was divided into three categories: A for candidates witl a marginally present or absent continuum, B for moderate continuum intensity objects, and $\mathrm{C}$ for candidates with a very strong, almost saturated continuum. Next in the classification is the estimate of the apparent equivalent width of the emission line. Candidates are designated 1,2 , or 3 according to the contrast of the emission line with respect to the continuum on the plates: the number 1 signifies a low-contrast emission line; and an equivalent width parameter of 3 is reserved for objects that exhibit a highly contrasted emission line, even saturated. Finally, we have used a concentration parameter which is based upon eye estimates of the widths of the spectra, perpendicular to the dispersion. Candidates for which the spectrum is clearly wider than stellar are denoted by "d" (for "diffuse"). No intermediate categories have been used because of the subjective nature of these eye estimates.

Information about the objects of the second list of our survey is presented in Table 2. Column (1) contains the positional UCM designation. Names composed of the values of right ascension and declination are given according to IAU rules; UCM stands for Universidad Complutense de Madrid. Objects are arranged in order of increasing right ascension. Equatorial coordinates for B1950.0 are given in columns (2) and (3). Column (4) con-

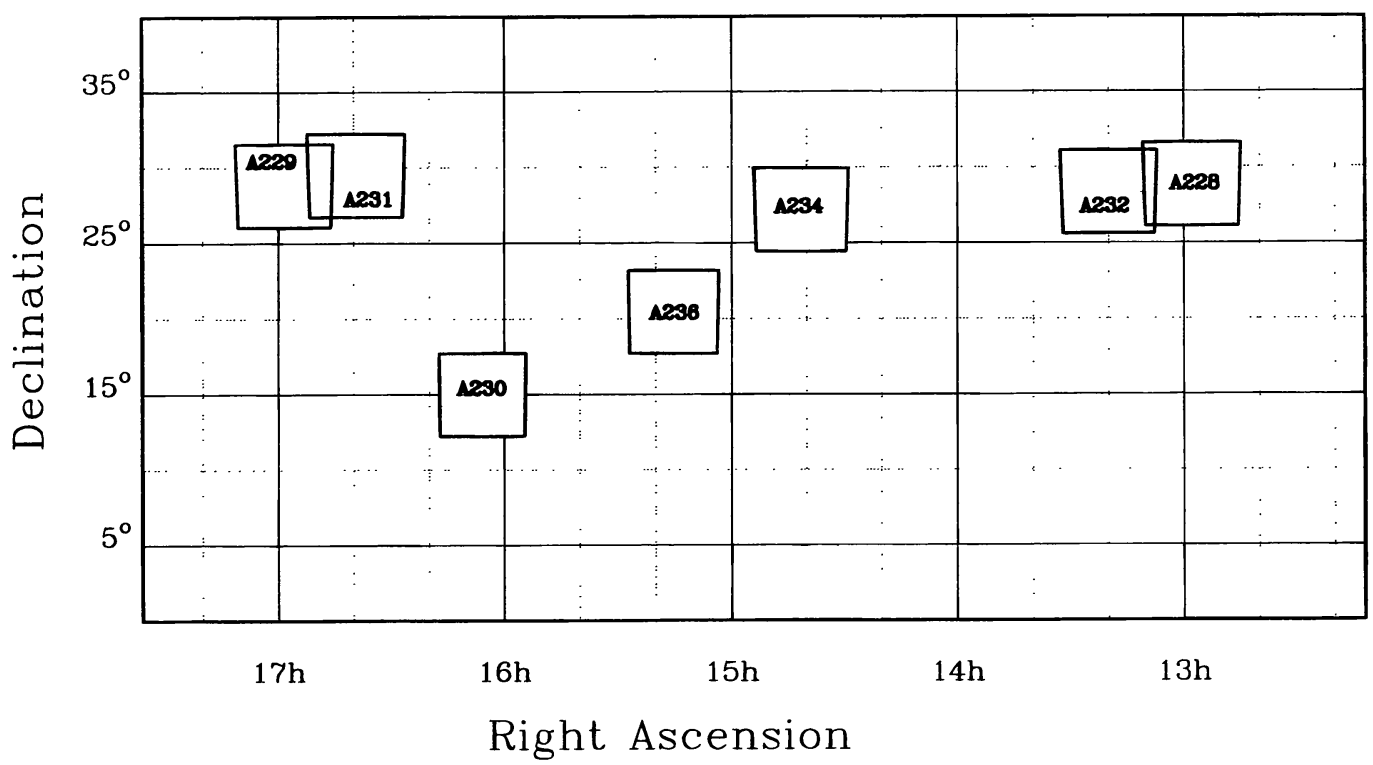

FIG. 1.-Schematic map of the sky showing the fields covered by the UCM survey in the 1987 June campaign and included in the second list. Each plate covers a field of $5.5 \times 5^{\circ} .5$. Field centers are provided in Table 1 . 
TABLE 2

A. UCM SURVEY LIST 2

\begin{tabular}{|c|c|c|c|c|c|c|c|c|}
\hline $\begin{array}{l}\text { UCM } \\
\text { (1) }\end{array}$ & $\begin{array}{l}\text { RA } 1950 \\
(2)\end{array}$ & $\begin{array}{c}\text { 0) } \mathrm{DEC} \\
(3)\end{array}$ & $\begin{array}{l}\text { Type } \\
(4)\end{array}$ & $\begin{array}{l}\mathbf{m} \\
(5)\end{array}$ & $\begin{array}{l}\text { size } \\
(6)\end{array}$ & $\begin{array}{l}\text { IRAS } \\
(7)\end{array}$ & $\begin{array}{c}\text { ZWG } \\
(8)\end{array}$ & $\begin{array}{c}\text { other names } \\
\text { (9) }\end{array}$ \\
\hline $1246+2727$ & 124634.8 & +272704 & A1d & 15.5 & $0.6 \times 0.3$ & & & $\begin{array}{l}\text { MK657 N4702 M+5-30-091 } \\
\text { KUG1246+274 }\end{array}$ \\
\hline $1247+2701$ & 124753.6 & +270121 & A1d & 16.0 & $0.4 \times 0.2$ & & & KUG1247+270 \\
\hline $1248+2912$ & 124836.0 & +291200 & B1d & 15.5 & $0.7 \times 0.5$ & $12485+2911$ & Z159.091 & N4735 M+05-30-104 KUG1248+291 \\
\hline $1253+2926$ & 125325.6 & +292612 & Al & & $0.2 \times 0.2$ & & & \\
\hline $1253+2756$ & 125340.6 & +275656 & B3 & 16.2 & $0.4 \times 0.3$ & & $\mathrm{Z} 160.020$ & $\begin{array}{l}\text { MK53 CG930 KUG1253+279 PB3129 } \\
\text { KUV12537+2757 }\end{array}$ \\
\hline $1254+2932$ & 125405.5 & +293235 & A1 & 17.1 & $0.2 \times 0.2$ & & & A2 213 \\
\hline $1254+2741$ & 125422.3 & +274131 & Ald & & $0.5 \times 0.2$ & & & \\
\hline $1254+2853$ & 125431.6 & +285339 & B1 & & $0.6 \times 0.3$ & & & \\
\hline $1254+2802$ & 125439.4 & +280238 & A1d & & $0.5 \times 0.3$ & & & \\
\hline $1254+2740$ & 125459.8 & +274030 & B2 & 16.3 & $0.4 \times 0.3$ & & & MK55 KUG1254+276 CG933 \\
\hline $1255+2819$ & 125532.7 & +281955 & Ald & 15.9 & $0.6 \times 0.4$ & & & M+05-031-035 CG934 KUG1255+283 \\
\hline $1255+3125$ & 125552.6 & +312546 & B2 & 15.5 & $0.6 \times 0.3$ & & & WAS064 CG936 M+05-31-033 \\
\hline $1255+2734$ & 125553.3 & +273452 & A2 & 16.5 & $0.4 \times 0.3$ & & & KUG1255+275 \\
\hline $1256+2717$ & 125601.8 & +271737 & A1 & & $0.3 \times 0.2$ & & & PB3168 \\
\hline $1256+2732$ & 125610.1 & +273206 & A2 & 16.2 & $0.5 \times 0.4$ & & $\mathrm{Z} 160.064$ & MK56 CG938 KUG1256+275 \\
\hline $1256+2701$ & 125614.5 & +270146 & A1 & & $0.7 \times 0.2$ & & & \\
\hline $1256+2910$ & 125618.6 & +291048 & A2d & & $0.6 \times 0.5$ & & & \\
\hline $1256+2823$ & 125637.3 & +282309 & B2 & 15.7 & $0.5 \times 0.4$ & $12566+2823$ & $\mathrm{Z160.213}$ & $\begin{array}{l}\text { N4858 M+05-31-051 CG942 } \\
\text { KUG1256+283 }\end{array}$ \\
\hline $1256+2754$ & 125640.2 & +275452 & B1 & 15.1 & $0.7 \times 0.6$ & & $\mathrm{Z160.073}$ & $\begin{array}{l}\text { MK58 M+05-31-057 CG943 } \\
\text { KUG1256+279 }\end{array}$ \\
\hline $1256+2722$ & 125651.5 & +272234 & B1 & & $0.4 \times 0.2$ & & & \\
\hline $1257+2754$ & 125702.8 & +275422 & A3 & 14.9 & $0.2 \times 0.2$ & & & PG1257+279 H4-1 PB3189 \\
\hline $1257+2826$ & 125712.6 & +282610 & A1d & & $0.3 \times 0.3$ & & & \\
\hline $1257+2808$ & 125740.3 & +280809 & A1 & 16.1 & $0.4 \times 0.3$ & & $\mathrm{Z} 160.243$ & MK60 CG949 KUG1257+281 \\
\hline $1258+2754$ & 125808.8 & +275427 & A2 & 15.7 & $0.6 \times 0.4$ & & $\mathrm{Z160.086}$ & CG953 KUG1258+279A \\
\hline $1259+2934$ & 125901.4 & +293458 & B3 & 13.9 & $0.3 \times 0.3$ & $12590+2934$ & Z160.096 & $\begin{array}{l}\text { N4922B U8135 M+05-31-099 } \\
\text { CG956 VV609B }\end{array}$ \\
\hline $1259+3011$ & 125920.1 & +301146 & B1d & & $0.4 \times 0.3$ & & & \\
\hline $1259+2755$ & 125943.3 & +275503 & B2 & 15.2 & $0.7 \times 0.5$ & $12596+2755$ & $\mathrm{Z160.106}$ & $\begin{array}{l}\text { N4926A M+05-31-107 } \\
\text { CG960 KUG1259+279 }\end{array}$ \\
\hline $1300+2907$ & 130001.6 & +290738 & A3 & 16.9 & $0.3 \times 0.2$ & & & CG963 PB3241 KUV13000+2908 \\
\hline $1300+3136$ & 130001.8 & +313629 & Ald & & $0.4 \times 0.4$ & & & \\
\hline $1300+2959$ & 130050.1 & +295930 & A2 & & $0.3 \times 0.2$ & & & PB3260 \\
\hline $1301+2904$ & 130158.9 & +290445 & A2 & 15.3 & $0.5 \times 0.5$ & & $\mathrm{Z} 160.128$ & KUG1301+290 \\
\hline $1302+2853$ & 130211.9 & +285346 & B1 & 16.0 & $0.4 \times 0.3$ & & & $\begin{array}{l}\text { CG968 KUG1302+288A } \\
\text { KUV13022+2854 }\end{array}$ \\
\hline $1302+3032$ & 130255.8 & +303244 & A1 & 16.5 & $0.4 \times 0.4$ & & & $\begin{array}{l}\text { MK62 CG971 KUG1302+305 } \\
\text { KUV13029+3033 PB3292 BA108 }\end{array}$ \\
\hline $1303+2908$ & 130321.8 & +290820 & A3d & 16.2 & $0.4 \times 0.3$ & & & CG972 PB3295 \\
\hline $1304+2808$ & 130412.6 & +280825 & A1d & 15.0 & $0.9 \times 0.5$ & $13042+2808$ & & $M+05-31-132$ KUG1304+281 \\
\hline $1304+2830$ & 130413.4 & +283008 & A2 & & $0.2 \times 0.2$ & & & \\
\hline $1304+2907$ & 130414.3 & +290705 & Ald & 15.1 & $0.9 \times 0.5$ & & $\mathrm{Z} 160.139$ & $\begin{array}{l}\text { M+05-31-133 KUG1304+291 CG974 } \\
\text { VV841 PB3313 }\end{array}$ \\
\hline $1304+2818$ & 130449.5 & +281852 & A1d & 15.7 & $0.5 \times 0.5$ & & $\mathrm{Z} 160.141$ & KUG1304+283 \\
\hline $1306+3100$ & 130610.5 & +310055 & Ald & & $0.7 \times 0.4$ & & & \\
\hline $\begin{array}{l}1306+2938 \\
1306+3111\end{array}$ & 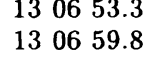 & $\begin{array}{l}+293803 \\
+311122\end{array}$ & $\begin{array}{l}\text { B2 } \\
\text { B1d }\end{array}$ & 15.1 & $\begin{array}{l}0.5 \times 0.4 \\
0.4 \times 0.4\end{array}$ & $13068+2938$ & $\mathrm{Z160.151}$ & $M+05-31-143$ CG980 KUG1306+296 \\
\hline $1307+2910$ & 130724.5 & +291025 & B1 & 13.9 & $1.5 \times 1.2$ & $13073+2910$ & $\mathrm{Z} 160.152$ & U08241 N5000 M+05-31-144 VV460 \\
\hline $1308+2958$ & 130825.3 & +295834 & A2d & 15.2 & $0.8 \times 0.6$ & & $\mathrm{Z} 160.155$ & N5004B IC4210 M+05-31-148 \\
\hline $1308+2950$ & 130839.3 & +295039 & B1 & 14.6 & $1.4 \times 0.6$ & $13086+2950$ & Z160.156 & U08259 N5004C M+05-31-150 \\
\hline $1309+2937$ & 130953.3 & +293705 & A1 & & $0.2 \times 0.2$ & & & \\
\hline $1310+2736$ & 131009.8 & +273640 & A1 & & $0.2 \times 0.2$ & & & \\
\hline $1310+3027$ & 131048.3 & +302725 & A1 & & $0.5 \times 0.3$ & & & \\
\hline $1312+3040$ & 131246.9 & +304007 & B1 & 15.4 & $0.7 \times 0.4$ & $13127+3040$ & $\mathrm{Z} 160.170$ & \\
\hline $1312+2954$ & 131247.6 & +295403 & A2d & & $0.6 \times 0.4$ & & & \\
\hline $1313+2938$ & 131342.2 & +293846 & A3 & 16.1 & $0.3 \times 0.2$ & & & WAS66 CG996 PB3449 TON151 \\
\hline $1314+2827$ & 131422.0 & +282751 & B1 & 16.3 & $0.4 \times 0.4$ & & & CG1001 \\
\hline $1320+2727$ & 132021.7 & +272734 & A2 & 17.0 & $0.3 \times 0.2$ & & & CG1019 \\
\hline $1321+2648$ & 132102.8 & +264817 & Ald & 15.6 & $0.7 \times 0.6$ & & $\mathrm{Z} 161.040$ & $M+05-32-012$ KUG1321+268 \\
\hline $1324+2926$ & 132404.9 & +292604 & A2 & 16.4 & $0.2 \times 0.2$ & & & WAS70 \\
\hline $1324+2651$ & 132430.1 & +265102 & $\mathrm{C} 2$ & 15.2 & $0.6 \times 0.4$ & $13244+2651$ & $\mathrm{Z} 161.052$ & MK454 M+05-32-020 KUG1324+268 \\
\hline $1325+2955$ & 132502.5 & +295529 & A1 & & $0.2 \times 0.2$ & & & \\
\hline $1330+3011$ & 133026.8 & +301127 & A1 & & $0.2 \times 0.2$ & & & \\
\hline $1331+2900$ & 133126.1 & +290034 & A3 & 17.4 & $0.1 \times 0.1$ & & & WAS74 \\
\hline $1428+2727$ & 142856.3 & +272730 & B3d & 15.5 & $0.7 \times 0.4$ & $14289+2727$ & $\mathrm{Z163.071}$ & MK685 M+05-34-061 KUG1428+274 \\
\hline $1429+2645$ & 142933.6 & +264553 & A2 & & $0.2 \times 0.2$ & & & \\
\hline $1430+2947$ & 143045.1 & +294739 & A3 & 17.0 & $0.4 \times 0.3$ & $14307+2947$ & & CG1239 \\
\hline
\end{tabular}


TABLE 2-Continued

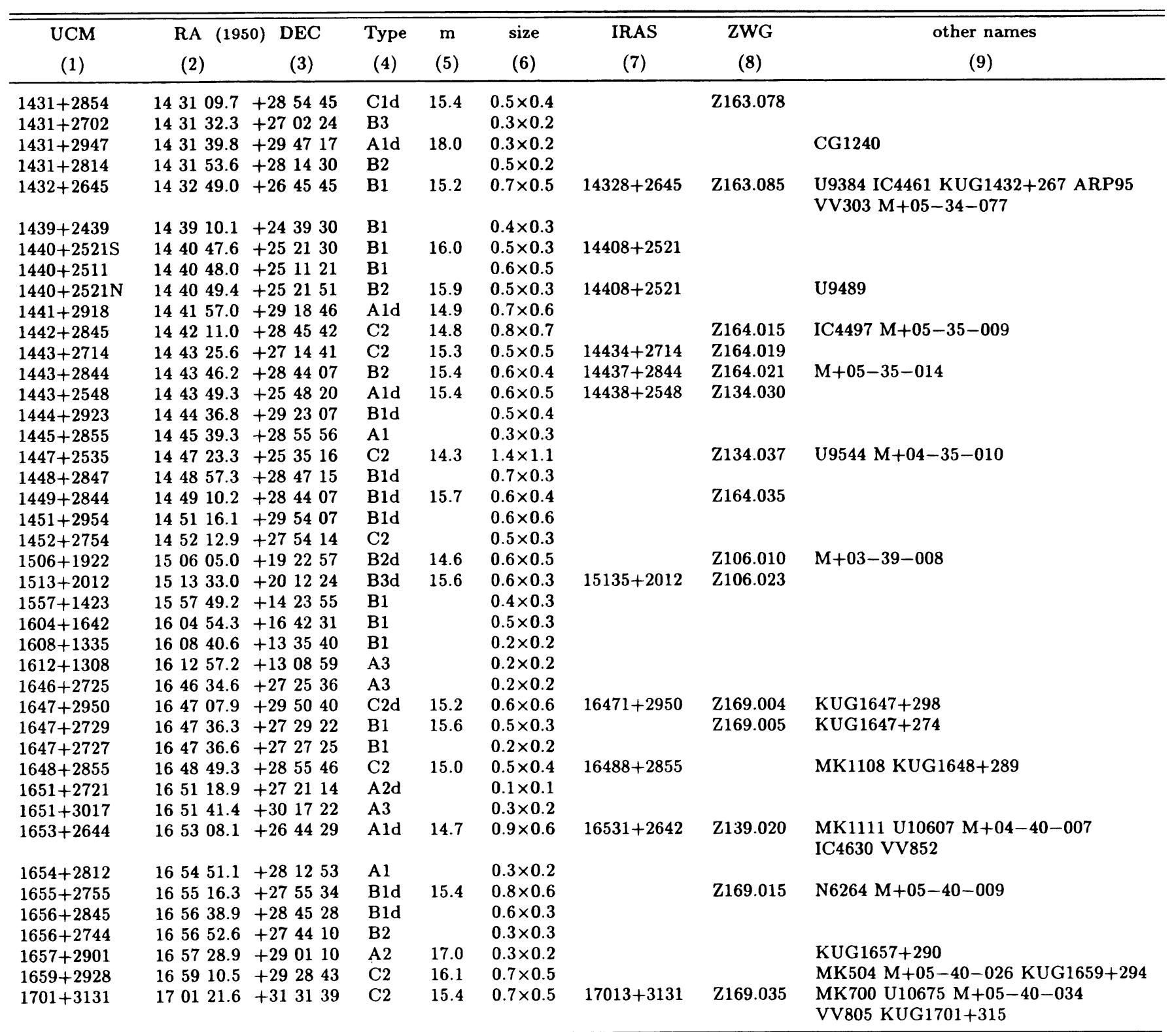

tains the object classification according to the threeparameter scheme described above. In column (5) the blue magnitudes are listed for previously cataloged galaxies. In column (6) we give object dimensions (major diameter $\times$ minor diameter) in minutes of arc. IRAS and Zwicky counterparts are given in columns (7) and (8). Finally, in column (9) previous designations are listed. The abbreviations refer to the following catalogs or surveys: A2 = Barbieri \& Rosino (1972), ARAK = Arakelian (1975), ARP = Arp (1966), CG = Case Low-Dispersion Northern Sky Survey (Pesch, Sanduleak, \& Stephenson 1991, and references therein), IC = Dreyer (1910), KARA $=$ Karachentsev (1971), $\quad$ KAZ $=$ Kazarian (Kazarian \& Kazarian 1980), KUG = Kiso (Takase \& Miyauchi-Isobe 1993, and references therein), $\mathbf{M}=\mathbf{M C G}$ (Vorontsov-Velyaminov, Arkipova, \& Kranogorskaja 1974, and references therein), $\mathbf{M K}=$ Markarian (Markarian 1967; Markarian, Lipovetskii, \& Stepanian 1981, and refer- ences therein), $\mathrm{N}=\mathrm{NGC}$ (Dreyer 1888), $\mathrm{PB}=$ PalomarBerger (Berger \& Fringant 1984, and references therein, TON = Tonanzintla (Iriarte \& Chavira 1957; Chavira 1957), U = UCG (Nilson 1973), WAS = Wasilewski (1983), and $Z=$ CGCG (Zwicky et al. 1961-1968; Zwicky 1971). A brief description of the appearance of the objects in the POSS prints and some additional information are given in the notes to the table. A comprehensive literature search has been made in order to check whether the candidates are objects not previously cataloged and to obtain previous designations for our survey objects. We have used the master list compiled by Dixon \& Sonnenborn (1980), the PGC (Paturel et al. 1989), and the NASA/IPAC Extragalactic Database NED).

We include identification charts for all the candidates of the survey in Figure 2 (Plates 18-23). Maps were copied from the Digitized Sky Survey, which is a digitized version of the Palomar Observatory Sky Survey (POSS) E plates 
PLATE 18

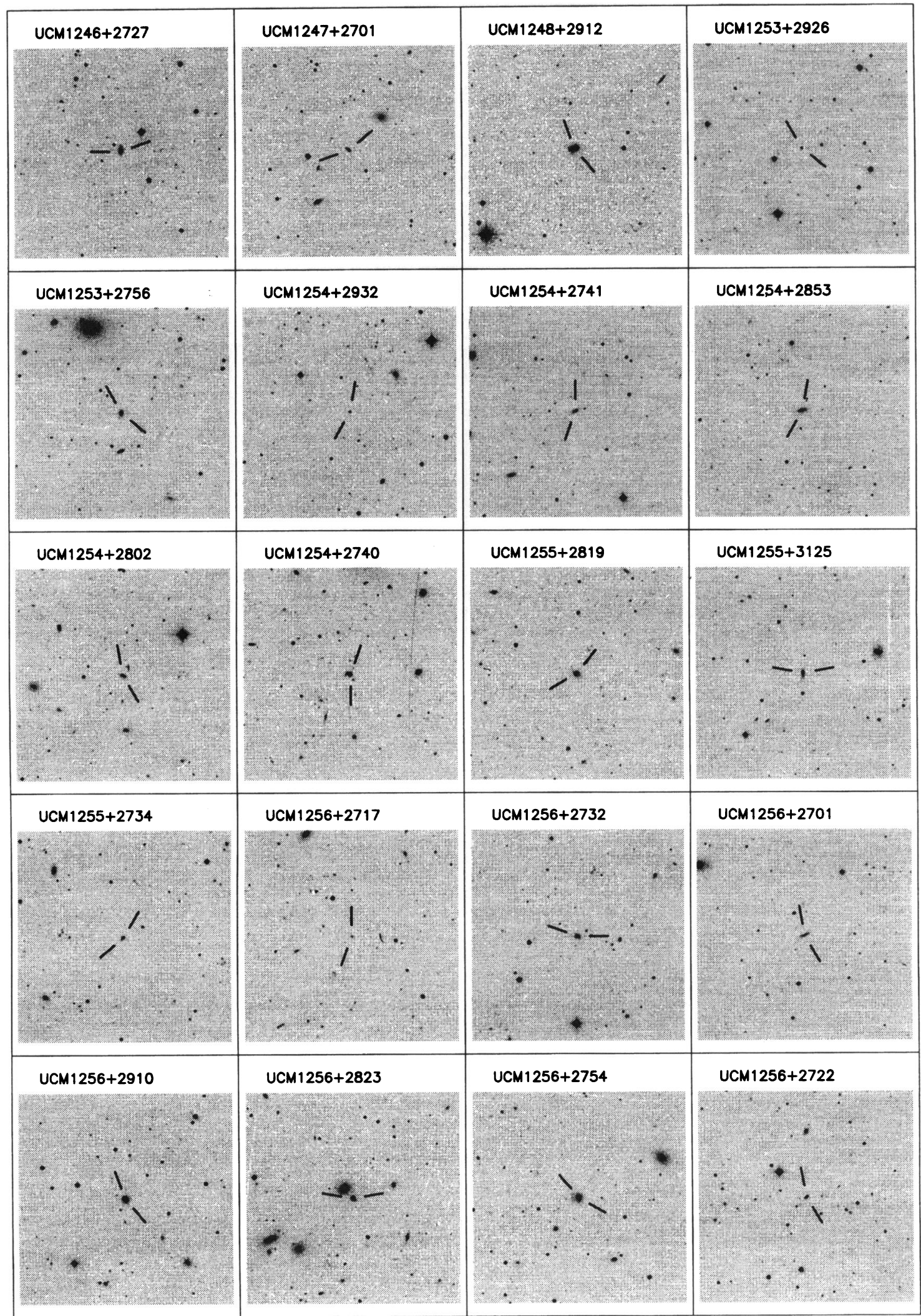

Fig. 2.-Finding charts for the objects found in the survey. North is up and east is to theleft. Each field covers a square $10^{\prime}$ wide. 


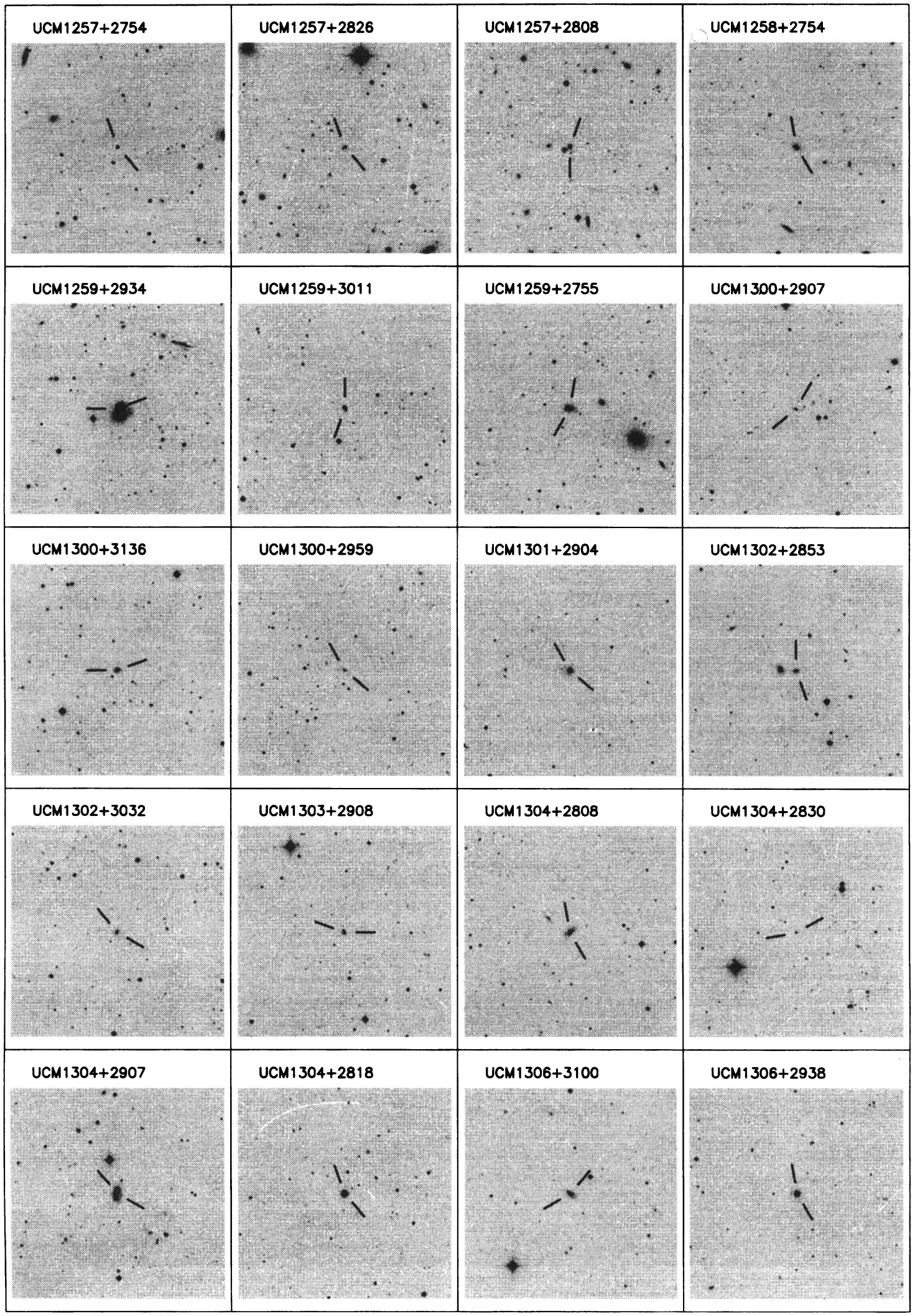

FIG. 2-Continued 
PLATE 20

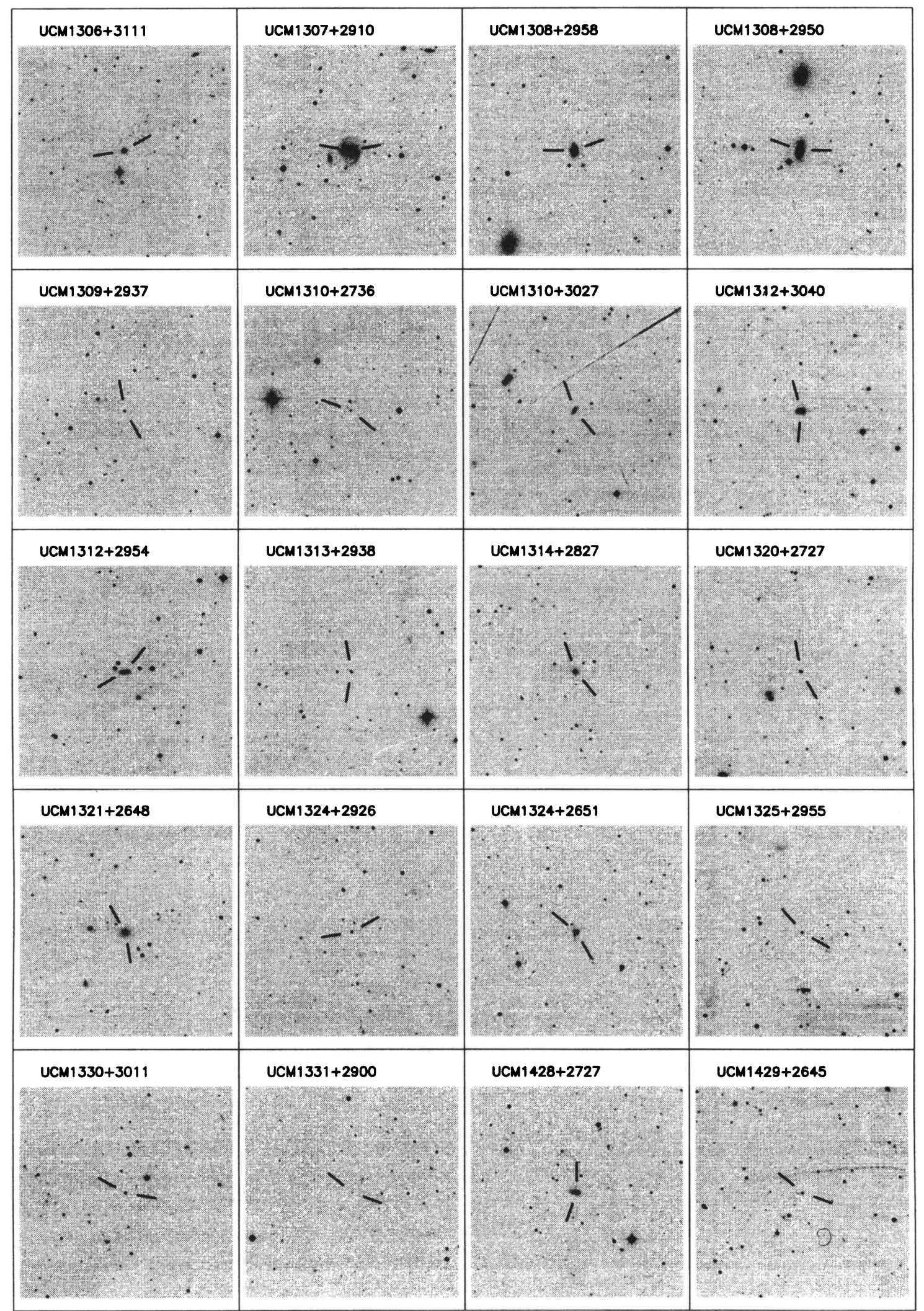

FIG. 2-Continued 


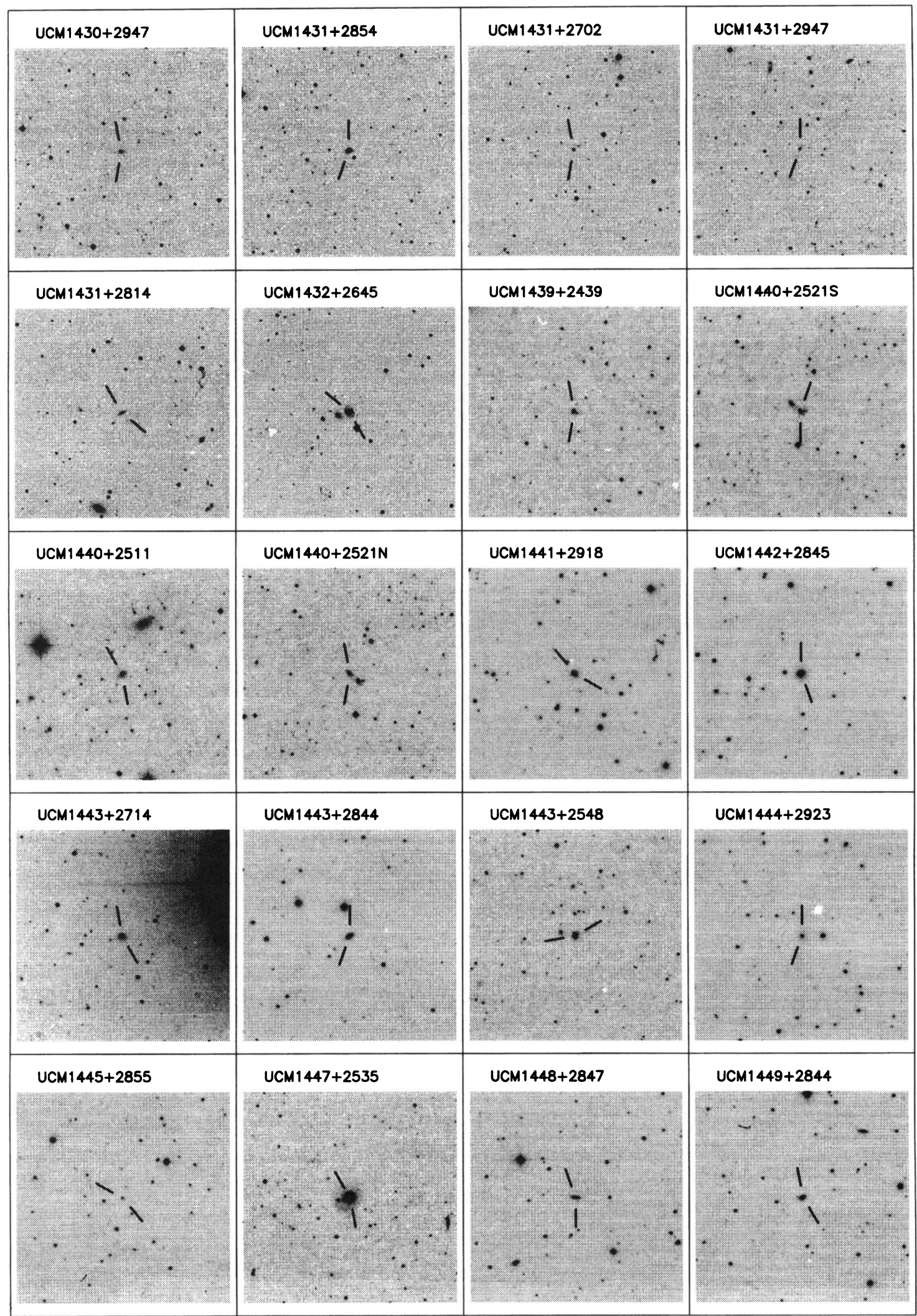

Fig. 2-Continued 
PLATE 22

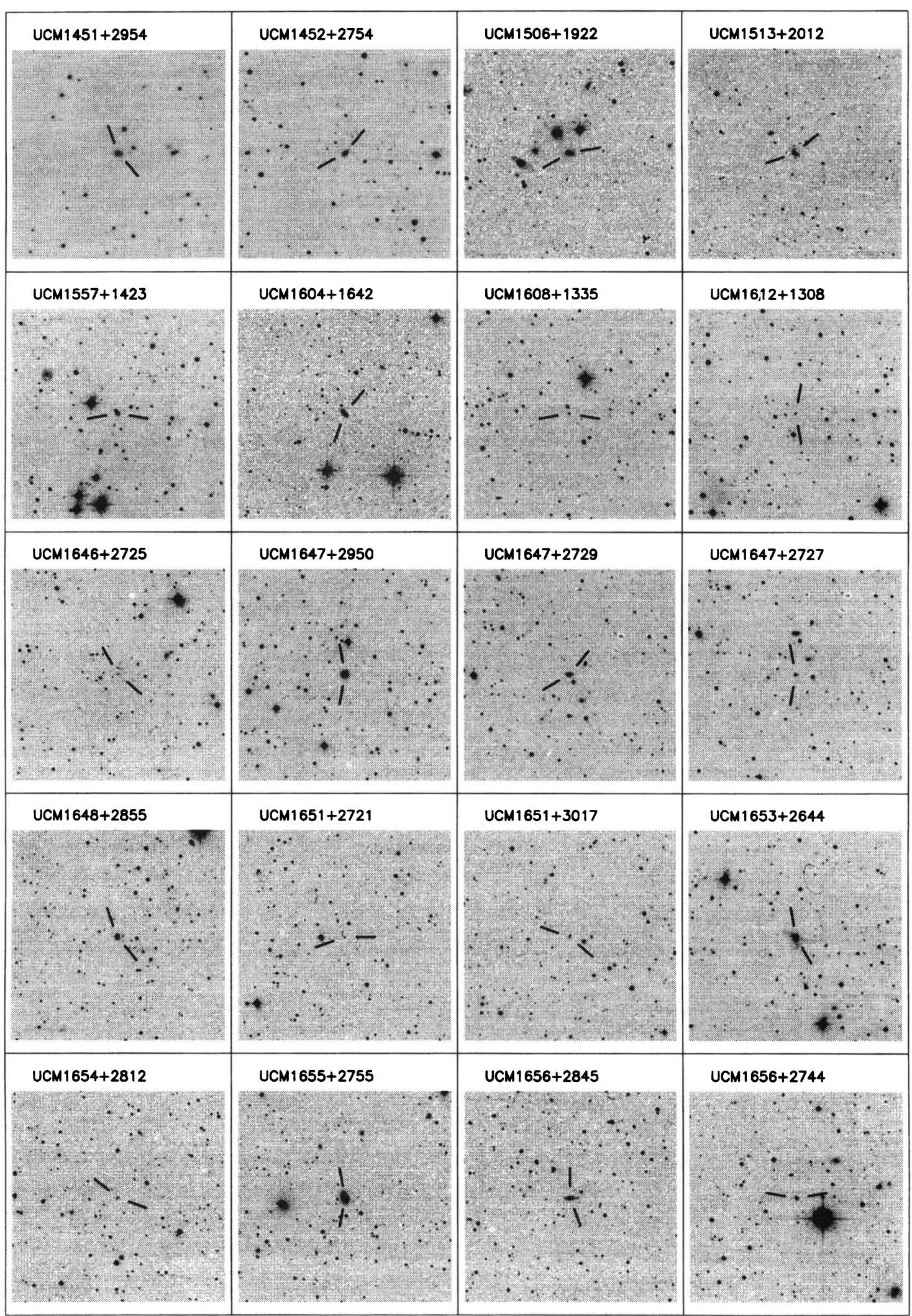

FIG. 2-Continued 


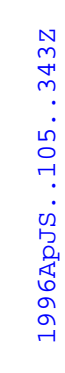

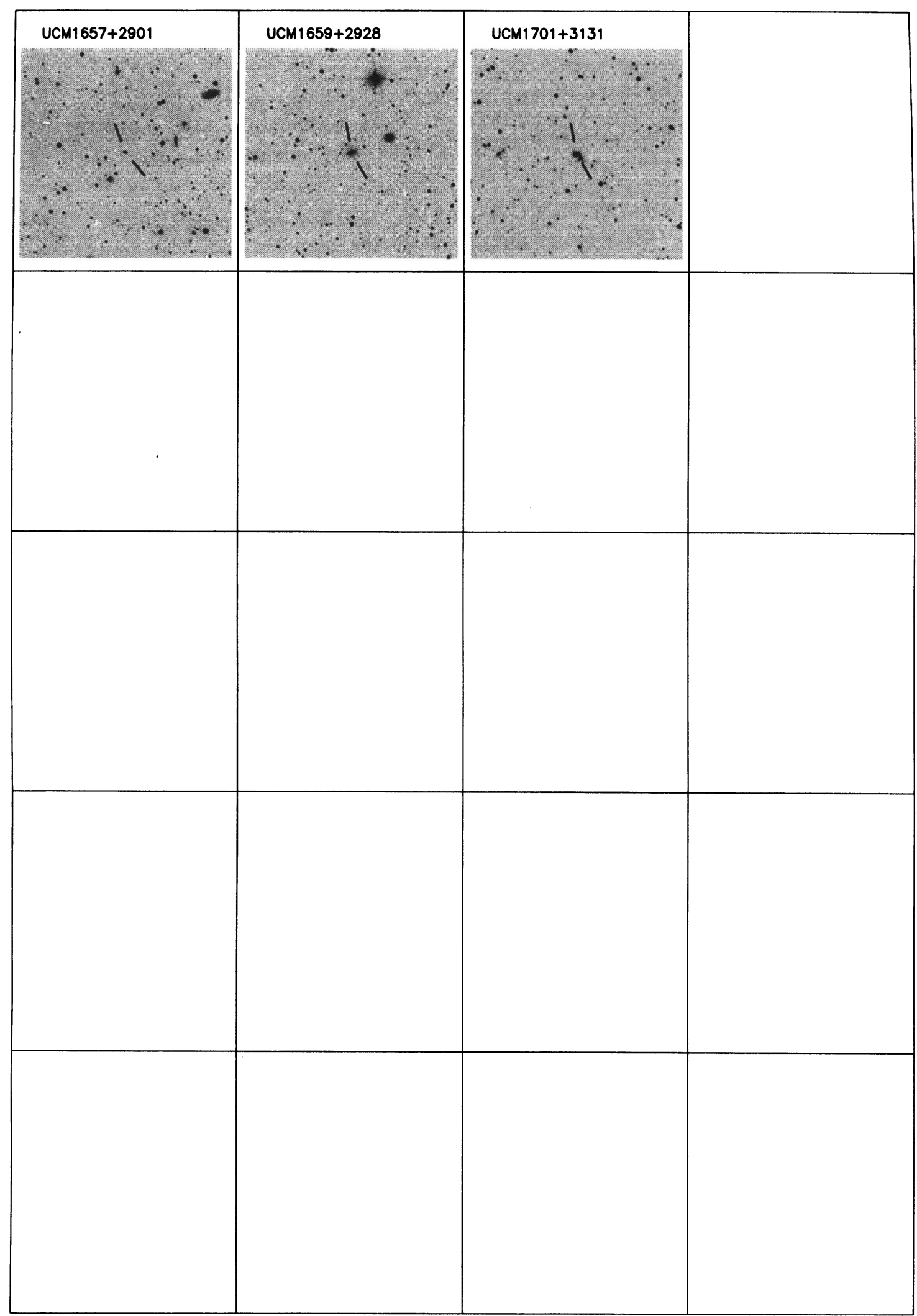

FIG. 2-Continued 
TABLE 2-Continued

B. Notes on Individual Objects

\begin{tabular}{|c|c|}
\hline $1246+2727$ irregular shape. & $1321+2648$ almost face-on spiral. \\
\hline $1247+2701$ almost edge-on spiral. & $1324+2926$ compact. \\
\hline $1248+2912$ almost face-on barred spiral. & $1324+2651$ two nuclei. A tail pointing to $S$. \\
\hline $1253+2926$ stellar-like appearance. & $1325+2955$ stellar-like appearance. \\
\hline $1253+2756$ oval. & $1330+3011$ compact oval. \\
\hline $1254+2932$ stellar-like appearance. & $1331+2900$ compact oval. \\
\hline $1254+2741$ spiral. & $1428+2727$ distorted spiral with knots at $\mathrm{S}$. \\
\hline $1254+2853$ spiral with bright nucleus. & $1429+2645$ compact. \\
\hline $1254+2802$ diffuse oval. & $1430+2947$ oval with bright nucleus. \\
\hline $1254+2740$ diffuse oval, western member of Mk55 pair. & $1431+2854$ diffuse spiral. \\
\hline $1255+2819$ diffuse with a knot at $S$ & $1431+2702$ compact. \\
\hline $1255+3125$ almost edge-on spiral, bright nucleus. & $1431+2947$ diffuse elongated. \\
\hline $1255+2734$ irregular shape. & $1431+2814$ almost edge-on spiral. \\
\hline $1256+2717$ diffuse oval. & $1432+2645$ barred spiral. \\
\hline $1256+2732$ spiral with bright nucleus. & $1439+2439$ spheroidal with a companion at SW. \\
\hline $1256+2701$ clumpy irregular. & $1440+2521 \mathrm{~S}$ southern spiral of a pair. \\
\hline $1256+2910$ face-on spiral. & $1440+2511$ spiral with bright nucleus. \\
\hline $1256+2823$ diffuse oval at SW of elliptical N4860. & $1440+2521 \mathrm{~N}$ northern spiral of a pair. \\
\hline $1256+2754$ spiral with bright nucleus. & $1441+2918$ spiral. \\
\hline $1256+2722$ spiral. & $1442+2845$ spheroidal. \\
\hline $1257+2754$ compact. & $1443+2714$ spiral with bright nucleus. Sy2. \\
\hline $1257+2826$ compact with a star at SE. & $1443+2844$ spiral with several knots. \\
\hline $1257+2808$ compact sourrounded by two galaxies. & $1443+2548$ spiral. \\
\hline $1258+2754$ distorted spiral. & $1444+2923$ compact spheroidal. \\
\hline $1259+2934$ distorted spiral. $\mathrm{N}$ member of system. Sy2. & $1445+2855$ compact oval. \\
\hline $1259+3011$ spiral with bright nucleus. & $1447+2535$ face-on spiral with knots. \\
\hline $1259+2755$ asymmetrical spiral. & $1448+2847$ edge-on spiral. \\
\hline $1300+2907$ distorted spiral. & $1449+2844$ spiral. \\
\hline $1300+3136$ diffuse oval. & $1451+2954$ oval diffuse. \\
\hline $1300+2959$ oval. & $1452+2754$ spiral. \\
\hline $1301+2904$ almost face-on spiral. & $1506+1922$ spiral with bright nucleus. \\
\hline $1302+2853$ oval. & $1513+2012$ distorted spiral. \\
\hline $1302+3032$ diffuse oval. & $1557+1423$ oval with a field star at NE. \\
\hline $1303+2908$ clumpy irregular. & $1604+1642$ spiral. \\
\hline $1304+2808$ spiral with extended nucleus. & $1608+1335$ stellar appearance. \\
\hline $1304+2830$ compact. & $1612+1308$ compact oval. \\
\hline $1304+2907$ clumpy irregular. & $1646+2725$ very elongated. \\
\hline $1304+2818$ face-on spiral. & $1647+2950$ oval diffuse. \\
\hline $1306+3100$ distorted spiral. & $1647+2729$ oval diffuse. \\
\hline $1306+2938$ diffuse oval. & $1647+2727$ spheroidal. Elongated companion at $\mathrm{W}$. \\
\hline $1306+3111$ oval. & $1648+2855$ diffuse spheroidal. \\
\hline $1307+2910$ face-on barred spiral. & $1651+2721$ stellar appearance. \\
\hline $1308+2958$ spiral. & $1651+3017$ oval with a field star at the NW. \\
\hline $1308+2950$ barred spiral. & $1653+2644$ distorted spiral. \\
\hline $1309+2937$ stellar-like appearance. & $1654+2812$ elongated. \\
\hline $1310+2736$ compact. & $1655+2755$ barred spiral. Sy2. \\
\hline $1310+3027$ spiral with bright nucleus. & $1656+2845$ almost edge-on spiral. \\
\hline $1312+3040$ ringed spiral. & $1656+2744$ oval with bright nucleus. \\
\hline $1312+2954$ almost edge-on spiral. & $1657+2901$ oval. \\
\hline $1313+2938$ oval with extension to the NE. & $1659+2928$ barred spiral with bright nucleus. Sy1. \\
\hline $1314+2827$ diffuse spheroidal. & $1701+3131$ spiral with a tail ponting to SW. Sy2. \\
\hline $1320+2727$ diffuse oval. & \\
\hline
\end{tabular}

produced by the Space Telescope Science Institute (STScI). These images were also used to derive object dimensions, which were obtained by fitting an ellipse to the points with density $3 \sigma$ over the background. Using the polynomial coefficients for the astrometric solution that appears in the headers of the individual image files, accurate coordinates of the objects were obtained with an accuracy of $1^{\prime \prime}$. This procedure is by far more precise than that employed to find the positions of the candidates of the first list. (Paper I), where uncertainties of $10^{\prime \prime}-20^{\prime \prime}$ were typical for new objects. This is 
TABLE 3

UCM SURVEY LIST 1 COORDINATES

\begin{tabular}{|c|c|c|c|c|c|c|c|c|}
\hline $\begin{array}{l}\text { UCM } \\
\text { Name }\end{array}$ & $\begin{array}{l}\text { RA (1950) } \\
\text { h m s }\end{array}$ & ) $\underset{0,1 /}{\mathrm{DEC}}$ & $\begin{array}{l}\text { UCM } \\
\text { Name }\end{array}$ & $\begin{array}{l}\text { RA (1950) } \\
\text { h m s }\end{array}$ & ) $\underset{0,11}{\mathrm{DEC}}$ & $\begin{array}{l}\text { UCM } \\
\text { Name }\end{array}$ & $\begin{array}{l}\text { RA (1950) } \\
\text { h m s }\end{array}$ & ) $\underset{0,1 "}{\mathrm{DEC}}$ \\
\hline $0000+2140$ & $\begin{array}{llll}00 & 00 & 35.7\end{array}$ & +214057 & $0054+2337$ & 005438.8 & +233711 & $2304+1640$ & 230426.2 & +164002 \\
\hline $0001+2024$ & 000134.6 & +202337 & $0056+0044$ & 005621.5 & +004407 & $2305+1621$ & 230459.9 & +162124 \\
\hline $0003+2200$ & $\begin{array}{lll}00 & 03 & 03.7\end{array}$ & +220015 & $0056+0043$ & $\begin{array}{llll}00 & 56 & 30.2\end{array}$ & +004353 & $2306+1703$ & 230622.8 & +170334 \\
\hline $0003+2215$ & $0003 \quad 18.0$ & +221527 & $0119+2156$ & $\begin{array}{llll}01 & 19 & 01.2\end{array}$ & +215654 & $2306+1947$ & $\begin{array}{lll}23 & 07 & 03.2\end{array}$ & +194744 \\
\hline $0003+1955$ & 000345.3 & +195529 & $0121+2137$ & 012153.6 & +213723 & $2307+2118$ & 230721.4 & +211901 \\
\hline $0005+1802$ & 000555.6 & +180245 & $0129+2109$ & $\begin{array}{llll}01 & 29 & 32.2\end{array}$ & +210912 & $2310+1800$ & 231009.8 & +180019 \\
\hline $0006+2332$ & 000620.0 & +233222 & $0130+2505$ & $\begin{array}{llll}01 & 30 & 33.4\end{array}$ & +250614 & $2312+2204$ & 231219.9 & +220403 \\
\hline $0009+2038^{a}$ & $\begin{array}{llll}00 & 09 & 49.9\end{array}$ & +203828 & $0134+2258$ & 013425.7 & +225756 & $2312+2500$ & $\begin{array}{lll}23 & 12 & 43.8\end{array}$ & +250034 \\
\hline $0012+2123^{b}$ & $0012 \quad 30.3$ & +212315 & $0135+2242$ & 013513.6 & +224204 & $2313+1842$ & 231309.7 & +184155 \\
\hline $0013+1944$ & $0013 \quad 14.6$ & +194208 & $0138+2016$ & 013758.3 & +201558 & $2313+2516$ & 231333.1 & +251701 \\
\hline $0014+1829$ & 001439.9 & +182938 & $0138+2047$ & 013814.8 & +204733 & $2315+1923$ & 231531.1 & +192332 \\
\hline $0014+1748$ & 001448.5 & +174824 & $0138+2216$ & $0138 \quad 15.6$ & +221648 & $2315+1658$ & 231544.1 & +165832 \\
\hline $0015+2212$ & 001531.8 & +221205 & $0139+2226$ & $\begin{array}{lll}01 & 39 & 10.9\end{array}$ & +222618 & $2316+2457$ & 231610.5 & +245735 \\
\hline $0017+1942$ & $\begin{array}{llll}00 & 17 & 21.7\end{array}$ & +194213 & $0141+2220$ & 014132.5 & +222005 & $2316+2459$ & 231612.5 & +245929 \\
\hline $0017+2148$ & $\begin{array}{llll}00 & 17 & 50.3\end{array}$ & +214841 & $0142+2137$ & $\begin{array}{lll}01 & 42 & 04.7\end{array}$ & +213755 & $2316+2028$ & 231658.6 & +202826 \\
\hline $0019+2045$ & $\begin{array}{llll}00 & 18 & 53.0\end{array}$ & +204624 & $0142+2441$ & $01 \quad 42 \quad 22.4$ & +244034 & $2317+2356$ & 231737.3 & +235650 \\
\hline $0018+2218$ & 001857.5 & +221857 & $0145+2519$ & 014459.8 & +251930 & $2319+2234$ & 231950.4 & +223412 \\
\hline $0018+2216$ & $\begin{array}{lll}00 & 18 & 57.3\end{array}$ & +221555 & $0147+2309$ & 014755.8 & +230907 & $2319+2243$ & 231951.9 & +224414 \\
\hline $0019+2201$ & $\begin{array}{lll}00 & 19 & 12.4\end{array}$ & +220129 & $0148+2124$ & 014818.5 & +212353 & $2320+2036$ & 232034.9 & +203605 \\
\hline $0022+2049$ & $00 \quad 22 \quad 07.4$ & +204928 & $0150+2032$ & 015055.9 & +203258 & $2320+2428$ & 232053.2 & +242833 \\
\hline $0023+1908$ & 002326.9 & +190834 & $0150+2056$ & 015100.0 & +205630 & $2321+2149$ & 232138.8 & +214940 \\
\hline $0034+2119$ & 003405.3 & +211957 & $0152+2039$ & 015205.0 & +203835 & $2321+2506$ & 232157.4 & +250639 \\
\hline $0036+2007$ & $\begin{array}{llll}00 & 36 & 19.0\end{array}$ & +200722 & $0155+2507$ & 015541.1 & +250657 & $2322+2204$ & $2322 \quad 40.9$ & +220459 \\
\hline $0037+2226$ & 003732.3 & +222629 & $0155+2223$ & 015542.3 & +222337 & $2322+2218$ & 232254.1 & +221811 \\
\hline $0038+0235$ & $00 \quad 38 \quad 17.4$ & +023404 & $0156+2410$ & 015627.2 & +241028 & $2323+2047$ & 232315.0 & +204737 \\
\hline $0038+2259$ & $0038 \quad 30.3$ & +225921 & $0157+2324$ & 015706.4 & +232409 & $2323+2252$ & 232356.7 & +225149 \\
\hline $0038+2302$ & 003845.7 & +230210 & $0157+2413$ & 015730.3 & +241355 & $2324+2448$ & 232410.8 & +244819 \\
\hline $0039+0054$ & $\begin{array}{lll}00 & 39 & 12.2\end{array}$ & +005412 & $0157+2102$ & 015745.7 & +210244 & $2325+2318$ & 232511.9 & +231849 \\
\hline $0040+0257$ & $\begin{array}{lll}00 & 40 & 02.9\end{array}$ & +025757 & $0159+2327$ & $\begin{array}{llll}01 & 59 & 00.8\end{array}$ & +232656 & $2325+2208$ & 232558.0 & +220845 \\
\hline $0040+2312$ & $\begin{array}{lll}00 & 40 & 10.0\end{array}$ & +231258 & $0158+2354$ & 015900.5 & +235444 & $2326+2435$ & 232619.1 & +243554 \\
\hline $0040+0220$ & 004015.6 & +022024 & $2238+2308$ & 223851.6 & +230844 & $2327+2154$ & 232737.2 & +215441 \\
\hline $0040-0023$ & 004054.1 & -002358 & $2239+2402$ & 223905.9 & +240241 & $2327+2515$ & 232740.4 & +251527 \\
\hline $0041+0134$ & 004122.1 & +013437 & $2239+1959$ & 223930.4 & +195959 & $2327+1956$ & 232759.7 & +195625 \\
\hline $0043+0245$ & 004309.9 & +024525 & $2241+2431$ & 224102.3 & +243115 & $2328+2109$ & 232827.9 & +210957 \\
\hline $0043+2440$ & $0043 \quad 27.9$ & +244206 & $2244+2049$ & 224406.5 & +204911 & $2329+2447$ & 232908.6 & +244737 \\
\hline $0043-0159$ & 004332.1 & -015945 & $2249+2149$ & 224932.1 & +214908 & $2329+2427$ & 232918.6 & +242732 \\
\hline $0044+2246$ & 004440.1 & +224634 & $2250+2427$ & 225010.0 & +242752 & $2329+2500$ & 232923.8 & +250109 \\
\hline $0045-0157$ & 004459.0 & -015742 & $2251+2352$ & $2251 \quad 18.9$ & +235214 & $2329+2511$ & 232936.1 & +251209 \\
\hline $0045+2256$ & 004504.2 & +225624 & $2251+2405$ & 225154.1 & +240444 & $2331+2214$ & 233150.0 & +221402 \\
\hline $0045+2206$ & $0045 \quad 17.2$ & +220602 & $2253+2219$ & 225305.4 & +222000 & $2333+2248$ & $\begin{array}{lll}23 & 33 & 02.9\end{array}$ & +224820 \\
\hline $0047+2051$ & $0047 \quad 16.0$ & +205109 & $2253+2453$ & 225318.8 & +245326 & $2333+2241$ & 233317.1 & +224104 \\
\hline $0047+2413$ & 004732.8 & +241332 & $2255+1930 \mathrm{~N}$ & 225508.8 & +193123 & $2333+2359$ & 233335.7 & +235922 \\
\hline $0047-0213$ & 004732.1 & -021324 & $2255+1930 \mathrm{~S}$ & 225507.9 & +193054 & $2334+2134$ & 233407.6 & +213418 \\
\hline $0047+2414$ & 004745.3 & +241454 & $2255+1926$ & 225516.7 & +192603 & $2344+2157$ & 234429.3 & +215712 \\
\hline 0049-0006 & 004913.6 & $-00 \quad 0637$ & $2255+1654$ & 225526.2 & +165402 & $2346+2011$ & 234651.3 & +201108 \\
\hline $0049+0017$ & $0049 \quad 15.6$ & +001735 & $2256+2002$ & 225622.8 & +200149 & $2348+2407$ & 234853.2 & +240731 \\
\hline $0049-0045$ & 004926.1 & -004529 & $2257+2438$ & 225707.4 & +243900 & $2351+2321$ & 235106.8 & +232116 \\
\hline $0049+0013$ & 004950.7 & +001325 & $2257+1606$ & 225750.5 & +160653 & $2352+2040$ & 235202.0 & +204055 \\
\hline $0050+0005$ & 005024.0 & +000552 & $2258+1920$ & 225839.5 & +192025 & $2352+2230$ & 235229.5 & +222945 \\
\hline $0050+2114$ & 005054.8 & +211432 & $2300+2014$ & 230049.6 & +201500 & $2353+2027$ & 235312.4 & +202721 \\
\hline $0051+2430$ & 005114.5 & +243005 & $2302+2053 \mathrm{~W}^{\mathrm{c}}$ & $\begin{array}{lll}23 & 02 & 56.1\end{array}$ & +205329 & $2354+2232$ & 235359.9 & +223050 \\
\hline $0053+2352$ & $0053 \quad 08.2$ & +235238 & $2302+2053 \mathrm{E}$ & 230259.8 & +205329 & $2357+2440$ & $2357 \quad 10.2$ & +244010 \\
\hline $0053-0049$ & $\begin{array}{lll}00 & 53 & 14.8\end{array}$ & -004915 & $2303+1856$ & $\begin{array}{lll}23 & 0307.6\end{array}$ & +185619 & $2357+2241$ & 235712.7 & +224144 \\
\hline
\end{tabular}

a Previous designation $0009+2024$

b Previous designation $0012+2109$.

c $2302+2053 \mathrm{~W}$ and E, previously known as $2302+2053$ and $2303+2053$.

why new coordinates have been derived for objects in that list. Table 3 presents the results.

\section{DISCUSSION}

The UCM survey has found up to now 264 candidates in 17 fields $\left(471.4 \mathrm{deg}^{2}\right)$. The overall density (candidates per square degree) is around 0.56, i.e., 6 times that of the Markarian survey (Mazarella \& Balzano 1986). Nearly half of the sample (138 candiates) are galaxies that do not appear in any published catalog. In Paper I some comparisons were made between the samples detected with different surveys and that obtained by us. In particular, comparisons were made with surveys carried out in the blue region, such as the University of Michigan survey (MacAlpine, Smith, \& Lewis 1977) and the Markarian Survey. The conclusions obtained when comparing these surveys with the UCM 
survey do not change when the new UCM fields surveyed are added to the comparison. The same is true for the comparison between UCM and IRAS samples (see also Gallego 1992; Rego et al. 1993).

\subsection{Comparison with the CGCG}

As stressed by Kinman (1984), it is of interest to note the ratio of galaxies with emission to the total number of galaxies in the field. The CGCG contains 1289 galaxies in the same area, 801 of which are galaxies with known redshift $z \leq 0.04$. Only 93 UCM candidates (35\% of the sample) are galaxies cataloged by Zwicky.

In Table 4 we show the results of a study of the ratio of Zwicky galaxies found with emission, obtained when the whole sample of UCM galaxies is considered. We have noticed that we derived a wrong value for the fraction of CGCG galaxies in the field covered by List 1 because of a fault in the selection procedure of the CGCG galaxies. These final results should be compared with those obtained by Kinman (1984) from a sample of CGCG galaxies in a 30 $\mathrm{deg}^{2}$ field near NGC 1023. He derived a ratio of galaxies with emission to the total number $24 \%$ (with the fraction rapidly dropping for fainter galaxies). The data have been extracted from NED and restricted to CGCG galaxies with known redshift $z \leq 0.04$. This table contains, for the Kinman survey, for our survey, for plate A228 (which contains the Coma Cluster of galaxies), and for the UCM survey when this region is not considered (field galaxies), the number of CGCG galaxies found in emission, the total number of CGCG galaxies, and the fraction of CGCG galaxies found as ELGs by our survey. For comparison purposes, the galaxies have been separated in the magnitude intervals used by Kinman (1984).

The UCM Survey finds about $15 \%$ of the CGCG galaxies, in the $471.4 \mathrm{deg}^{2}$ surveyed, to be ELGs, when galaxies outside the Coma Cluster are considered. While this fraction is very dependent on the magnitude interval for the Kinman survey, we have found an almost constant ratio. The Kinman survey was based on plates taken at $400 \AA$ $\mathrm{mm}^{-1}$, and the spectra are 5 times more dispersed than those registered in our plates. Since the spectra are spread out, the emission-line visibility improves for the brightest galaxies with the penalty of a brighter limiting magnitude. Moss \& Whittle (1993) found 77 out of 201 (38\%) of Zwicky galaxies with $\mathrm{H} \alpha$ emission, when surveying galaxies belonging to clusters. Since these authors considered only spiral galaxies, it is not a surprise that this ratio was higher than the value obtained by Kinman with tle same instrumentation.

Plate A228 has been separated in order to find any possible difference in this fraction when surveying a cluster of galaxies. The fraction is significantly lower $(7 \%)$ than the value derived for field galaxies $(15 \%)$. It is interesting to note the paucity of bright galaxies with emission found by us in plate A228: only $3 \%$ of those with $m_{z}<15.0$, but $9 \%$ for $m_{z} \geq 15.0$. To explore this problem further, we have used the data of Doi et al. (1995), who have made a catalog of galaxies in the Coma Cluster using automated surface photometry. The percentages of late-type galaxies in the Coma Cluster for galaxies brighter and fainter than 15.0 are $42 \%$ and $48 \%$, respectively. This rules out possible explanations based on the absence of disk galaxies or on the morphological distribution of magnitudes. The spirals that are located within a radius of $1^{\circ}$ from the cluster center are severely depleted in $\mathrm{H}$ I with respect to the outer spirals (Bothun, Schommer, \& Sullivan 1984). Since the ELGs should be gas rich, the existence in the Coma Cluster of such a strong radial gradient in $\mathrm{H} \mathrm{I}$ content of its spiral galaxies could be the cause of this low fraction of ELGs. This hypothesis is supported by the result that UCM galaxies in the Coma Cluster are less clustered that the spirals of this cluster (Gallego et al. 1996).

On the other hand, plate A228 contains 46 UCM candidates. These UCM objects, which were not cataloged by Zwicky, are, on average, fainter than CGCG galaxies, because our survey is deeper than the Zwicky one. Using our photometric data in the Gunn-Thuan $r$ band (Vitores et al. 1995), mean values of $r=14.7 \pm 0.7$ and $r=15.9 \pm 0.7$ are found for $15 \mathrm{UCM}$ objects that are CGCG galaxies and for $26 \mathrm{UCM}$ galaxies not cataloged by Zwicky, respectively.

We conclude that our survey is losing an important fraction of bright and well-known galaxies whose lowresolution spectra appear saturated in the prism plates. Our survey is more biased toward fainter galaxies than that carried out by Kinman. Although the comparison has been made with the Zwicky catalog as reference and our survey has a fainter limiting magnitude, from the sample of the UCM survey, a smaller ratio has been found in a cluster environment $(7 \%)$ than in the field $(15 \%)$ for the galaxies with emission.

\subsection{Comparison with the KUG Survey}

The KUG survey (Kiso ultraviolet-excess galaxies) has found up to now 8162 galaxies in 170 fields covering some $5100 \mathrm{deg}^{2}$, yielding a number density of 1.8 KUGs per square degree (Takase \& Miyauchi-Isobe 1993). Our survey has covered some fields in common with the KUG survey. In order to perform a comparison, we have restricted our discussion to the six fields that completely overlap with ours. The results are summarized in Table 5. For each field listed in the first column, the total number of CGCG galaxies is given in column (2), the numbers of KUGs and

TABLE 4

Fraction of CGCG Galaxies Found with Emission

\begin{tabular}{|c|c|c|c|c|c|c|c|c|c|c|c|c|}
\hline \multirow{2}{*}{$\begin{array}{r}\begin{array}{c}\text { Magnitude } \\
\text { interval }\end{array} \\
<13.0\end{array}$} & \multicolumn{3}{|c|}{$\begin{array}{l}\text { Kinman } \\
\text { Survey }\end{array}$} & \multicolumn{3}{|c|}{$\begin{array}{c}\text { UCM } \\
\text { List } 1 \& 2\end{array}$} & \multicolumn{3}{|c|}{$\begin{array}{c}\text { Plate A228 } \\
\text { (Coma Cluster) }\end{array}$} & \multicolumn{3}{|c|}{$\begin{array}{l}\text { UCM List } 1 \& 2 \\
\text { without A228 }\end{array}$} \\
\hline & 5 & 6 & $(83$ & 1 & 19 & $(5 \%)$ & 0 & 3 & $(0 \%)$ & 1 & 16 & $(6 \%)$ \\
\hline $13.0-13.9$ & 4 & 8 & $(50 \%)$ & 7 & 72 & $(10 \%)$ & 2 & 14 & $(14 \%)$ & 5 & 58 & $(9 \%)$ \\
\hline $14.0-14.9$ & 8 & 22 & $(36 \%)$ & 23 & 183 & $(13 \%)$ & 0 & 48 & $(0 \%)$ & 23 & 135 & $(17 \%)$ \\
\hline $15.0-15.6$ & 7 & 44 & $(16 \%)$ & 46 & 353 & $(18 \%)$ & 8 & 112 & $(7 \%)$ & 38 & 241 & $(16 \%)$ \\
\hline$>15.6$ & 2 & 30 & $(7 \%)$ & 17 & 121 & $(14 \%)$ & 6 & 50 & $(12 \%)$ & 11 & 71 & $(16 \%)$ \\
\hline total & 26 & 110 & $(24 \%)$ & 94 & 748 & $(13 \%)$ & 16 & 227 & $(7 \%)$ & 78 & 521 & $(15 \%)$ \\
\hline
\end{tabular}


TABLE 5

Comparison WITH the KUGs SURveY

\begin{tabular}{lrrrr}
\hline \hline Plate & CGCG & \multicolumn{1}{c}{ KUG } & UCM & KUG \\
& & \multicolumn{1}{c}{ (MK) } & \multicolumn{1}{c}{ (MK) } & UCM \\
\hline A194 & 72 & $44(1)$ & $34(3)$ & 6 \\
A197 & 41 & $60(1)$ & $14(1)$ & 4 \\
A200 & 42 & $26(3)$ & $12(2)$ & 4 \\
A205 & 70 & $22(1)$ & $21(1)$ & 2 \\
A228 & 253 & $101(5)$ & $46(7)$ & 20 \\
A232 & 63 & $36(3)$ & $12(1)$ & 1 \\
total & 541 & $289(14)$ & $139(15)$ & 37 \\
\hline
\end{tabular}

UCM galaxies are listed in columns (3) and (4), respectively, and the number of KUGs that are also UCM galaxies is given in column (5). In columns (4) and (5) the number of galaxies that are also Markarian is given in parentheses and can be used to stress the difference in steepness and composition between Markarian and UCM or KUG samples. The summary, tabulated in the last row, shows some interesting results. The mean surface density of the whole KUG survey is 3 times that of the UCM survey. However, the KUGs in these fields outnumber the UCM galaxies only by a factor of 2. This local difference in density should be ascribed to the spatial distribution of galaxies in this region of the sky; i.e., a greater fraction of low-redshift galaxies. It is worth remembering that a color survey such as KUG is not limited by redshift as the UCM is. We expect that a fraction of the KUGs have a redshift that prevents detection by us. For comparison, Comte et al. (1994) found $25 \%$ of KUGs with $z>0.04$ (34 out of 136) in a different region of the sky. The number of common galaxies is rather small: 37 out of 139 UCM galaxies, i.e., $27 \%$. This points to a difference in the population derived from both surveys. A detailed comparison has been made using the spectroscopic observations of our whole sample and the published spectroscopic data on KUGs (Comte et al. 1994) confirming this result (Gallego et al. 1996).

The relationship expected between the KUG UV-excess parameter and the equivalent width of $\mathrm{H} \alpha$ in the UCM galaxies has been found to have values similar to those quoted by Comte et al. (1994). The median $\operatorname{EW}(\mathrm{H} \alpha)$ is 47 , 61, and $96 \AA$ for low (L), medium (M), and high (H) UVexcess KUGs, respectively. We note, however, the existence of two outliers: UCM $2258+1920$ classified as L in the Kiso survey, with $\mathrm{EW}(\mathrm{H} \alpha)=176 \AA$, and UCM $0003+1955$ with $\mathrm{EW}(\mathrm{H} \alpha)=348 \AA$ and classified as M. The first outlier is a typical case of a galaxy easily recovered by our survey and difficult to find with surveys looking for UV-excess or blue lines, i.e., high extinction $\left(E_{B-V}=0.35\right)$ and $\mathrm{EW}(\mathrm{H} \beta) \sim 10 \AA$. The explanation for the high value of $\mathrm{EW}(\mathrm{H} \alpha)$ found in the second galaxy is straightforward: it is Mrk 335, a well-known Seyfert 1.

\subsection{Comparison with the Case Survey}

A similar result is obtained when comparing our survey with the Case Low-Dispersion Northern Sky Survey (Pesch et al., and references therein). A small portion of Plate A234 and a region of Plates A228 and A232 are covered by the Case list of Paper XIII (Stephenson, Pesch, \& MacConnell 1992) and Paper XI (Sanduleak \& Pesch 1990), respectively. A search in NED yields 56 CG objects, 17 of them also found by our survey, which in total selects 33 ELGs in the common region in field A228. It should be remembered that the Case survey detects galaxies with UV-excess spectra and/or blue lines. In the original lists of Case, 37 of the 39 galaxies not detected by our survey are classified as " $\mathrm{mb}$ " (moderately) without apparent lines, and only two have suspected emission lines. It is difficult to know which fraction of these " $\mathrm{mb}$ " Case galaxies really do have emission lines in their spectra. Salzer et al. (1995) present spectroscopic observations for blue and/or emission-line galaxies from the Case survey. Using their data, it is found that there are only five of the 63 Case galaxies classified as " $\mathrm{mb}$ " without lines that show spectra with $\mathrm{EW}(\mathrm{H} \beta)>10 \AA$. From this result we expect that most of the Case galaxies not detected by us are star-forming galaxies with a very low level of activity. We have measured high values for the equivalent width of the $\mathrm{H} \alpha+[\mathrm{N} \mathrm{II}]$ blend of CGs that are also UCM galaxies, ranging from 45 to $353 \AA$, with a mean value of $108 \AA$.

It is interesting to note that the Case survey seems to be able to select some galaxies with low-excitation spectra. Some authors (Salzer 1989) claim that these galaxies can be recovered by a blue objective-prism survey by the presence of the [O II] $3727 \AA$ line or by their blue continua. Weistrop $\&$ Downes (1991) selected all galaxies with $m_{B} \leq 16$ for which emission lines were detected on the objective-prism spectra from the third Case list. The follow-up spectroscopic observations showed that 11 out of 32 ELGs exhibit low-excitation spectra (see, for instance, the spectrum of CG 279 in Fig. $1 b$ of their paper). As they did not list the fluxes for [O II] $3727 \AA$, we assume that this line is very weak for these galaxies and that they have been detected by their blue color. The Case survey selects blue galaxies as those that they find to display continua shortward of $4000 \AA$ much more strongly than are seen in an average galactic spectrum, and one out of every 10 field galaxies on their plates meets this definition. Moreover, in the third Case list these galaxies are classified as " $\mathrm{mb}$ " or moderately blue continuum and without lines, and only CG 220 has been classified as " $w$ ?," indicating the uncertainty of the presence of the [O II] $3737 \AA$ line. Our results support the conclusions of Salzer et al. (1995), who conclude that the dual selection method is better able to detect galaxies at lower levels of activity than most previous surveys.

On the other hand, there are $15 \mathrm{UCMs}$ without a CG counterpart. As expected, most of them have low-excitation spectra with absent or very weak lines in the blue part of the spectrum (Case plates have a spectral range from $~ 3300$ to $\sim 5350 \AA$ ). However, we have found six ELGs that have [O II $] 3727 \AA, \mathrm{H} \beta$, or $[\mathrm{O}$ III $] 4959,5007 \AA$ lines with equivalent widths in excess of $20 \AA$. We cannot find any explanation of why these objects have not been found by the Case survey. Two of them have very bright blue lines and a rather weak continuum: UCM $1304+2830$, a compact H II galaxy, and UCM $1257+2754$, a planetary nebula. Since only three of them are KUGs, this leaves 12 out of the 33 UCM galaxies (i.e., $36 \%$ ) without a CG or KUG counterpart. An important conclusion can be drawn from the previous discussion: a significant fraction of low-ionization star-forming galaxies are not selected by the objective-prism surveys carried out in the blue, neither by the [O II] $3727 \AA$ emission line nor by their blue colors.

It is well established that surveys based on different techniques select samples of ELGs that are not identical. With the results presented here, it is clear that only an objective- 
prism survey that selects galaxies by the presence of emission lines, both in the blue and $\mathrm{H} \alpha$ and blue or UV-excess color, will yield a less biased sample of ELGs. The prototype of this kind of survey is the Second Byurakan Spectral Sky Survey (SBS) (Markarian, Stepanian, \& Erastova 1987), which observes each field in three colors: blue and UV region (IIIa-J + 1.5 prism), green region (IIIa-J + GG495 + $3^{\circ}$ prism), and red spectral range (IIIa-F + RG2 $+4^{\circ}$ prism).

\section{SUMMARY}

In this paper we have presented the UCM survey List 2. A compilation of descriptions and positions is presented for 103 suspected emission-line objects. The second list covers $201.4 \mathrm{deg}^{2}$ in seven fields. The total number of UCM candidates, considering the two published lists, amounts to 264 candidates found in 17 fields covering $471.4 \mathrm{deg}^{2}$. The overall density (candidates per square degree) is 0.56 , i.e., 6 times that of the Markarian survey.

Nearly half of the sample (138 candidates) are galaxies that do not appear in any published catalog. Only $93 \mathrm{UCM}$ candidates (35\% of the sample) are galaxies cataloged by Zwicky. On the other hand, the fraction of CGCG galaxies found with emission by the UCM survey is $15 \%$ for field galaxies with known redshift $z \leq 0.04$. We are not claiming that this is the actual fraction of galaxies with emission lines in their spectra. It should be noted that our survey is also limited by the total flux and the equivalent width of the $\mathrm{H} \alpha+[\mathrm{N} \mathrm{II}]$ blend $(\sim 20 \AA)$, the red magnitude (continuum flux around $\mathrm{H} \alpha$ ), and surface brightness. This is why we are losing an important fraction of bright CGCG galaxies whose spectra appear saturated on the PO plates. Some other CGCG galaxies have $\mathrm{EW}(\mathrm{H} \alpha+[\mathrm{N} \mathrm{II}] \leq 20 \AA$, and their low-resolution spectra will appear without noticeable emission lines on the prism plates. These galaxies are easily selected by the survey of Kinman, who employed a PO setup that provided spectra 5 times more dispersed. However, the limiting magnitude of our survey is fainter than that of Kinman, and most of our galaxies are selected from those not classified by Zwicky $(65 \%)$.

Using the Zwicky catalog as a reference, from the sample of the UCM survey a smaller ratio has been found in the Coma Cluster $(7 \%)$ than in the field $(15 \%)$ for the galaxies with emission. This means that the fraction of emission-line galaxies in this cluster environment is around half that of the field. The density of UCM galaxies in Plate A228, covering the Coma Cluster, is $~ 1.5$ ELGs per square degree, while this number reduces to $\sim 0.5$ for the remainder of the survey (field galaxies). This result is not surprising because of the high density of galaxies of any kind in the cluster. However, the relative number of UCM and CGCG galaxies with $z \leq 0.04$ in both regions is 0.4 (five CGCG galaxies for each UCM galaxy) for the field and 0.2 for the Coma Cluster. We have again obtained a factor of 2 between the fraction of emission-line galaxies in the field and a cluster environment.

We have shown that our survey is complementary to other surveys carried out with different techniques. In particular, the UCM survey is able to find new emission-line objects in areas covered by other surveys, including those with dual selection methods, i.e., UV-excess and blue emission-line, such as the Kiso and the Case LowDispersion Northern Sky surveys. This means that each field should be surveyed with different techniques to recover a more representative and less biased sample of ELGs.

It is our great pleasure to express thanks for the friendly assistance and observing support received from the Calar Alto Observatory staff especially from Kurt Birkle. A merged copy of the KUG catalog (Vols. 1-17) was kindly provided by Nagako Miyauichi-Isobe in computerreadable format. Thanks to Jessica Rosenberg for sending us a preprint with the data for galaxies from List 1 and 2 of the Case survey. We also express our thanks to our colleagues at the Departamento de Astrofísica for their encouraging support and many fruitful discussions. We wish to thank the anonymous referee for useful comments that improved this paper.

The finding charts were copied from The Digitized Sky Survey. The images are based on photographic data obtained using the Oschin Schmidt telescope on Palomar Mountain. The Palomar Observatory Sky Survey was funded by the National Geographic Society. The Oschin Schmidt telescope is operated by the California Institute of Astronomy and the Palomar Observatory. The Digitized Sky Survey was produced at the Space Telescope Science Institute (STScI) under US Government grant NAG W-2166.

This research has made use of the NASA/IPAC Extragalactic Database (NED), which is operated by the Jet Propulsion Laboratory, Caltech, under contract with the National Aeronautics and Space Administration.

This work was supported in part by the Spanish Programa Sectorial de Promoción General del Conocimiento under grant PB93-456.

\section{REFERENCES}

Alonso, O., Zamorano, J., Regom M., \& Gallego, J. 1995, A\&A, 113, 399 Alonso-Herrero, A., Aragón-Salamanca, A., Zamorano, J., \& Rego, M. 1996, MNRAS, 278, 412

Arakelian, M. A. 1975, Soob. Byurakan Obs., 47, 1

Arp, H. C. 1966, Atlas of Peculiar Galaxies (Pasadena: Caltech)

Barbieri, C. \& Rosino, L 1972, Ap\&SS, 16, 324

Berger, J., \& Fringant, A.-M. 1984, A\&AS, 58, 565

Birkle, K. 1984, in IAU Colloq. 78, Astronomy with Schmidt-Type Telescopes, ed. M. Capaccioli (Dordrecht: Reidel), 203

Bothun, G. D., Schommer, R. A., \& Sulivan III, W. T. 1984, AJ, 89, 466

Chavira, E. 1957, Bol. Obs. Tonantzintla Tacubaya, 2, 18, 3

Comte, G., Augarde, R., Chalabaev, A., Kunth, D., \& Maehara, H. 1994, A\&A, 285, 1

Dixon, R. S., \& Sonnenborn, G. 1980, A Master List of Nonstellar Optical Astronomical Objects (Columbus: Ohio State Univ. Press)

Dreyer, J. L. E. 1988, MmRAS, 49, 1

1910, MmRAS, 59, 105

Doi, M., Fukugita, M., Okamura, S., \& Tarusawa, K. 1995, ApJS, 97, 77
Gallego, J., Zamorano, J., Rego, M., Alonso, O., \& Vitores, A. G. 1996, A\&A, in press

Gallego, J., Zamorano, J., Rego, M., \& Vitores, A. G. 1994, A\&A, 290, 705 Guibert, J., \& Moreau, O. 1991, Messenger, 64, 69

Iriarte, B., \& Chavira, E. 1957, Bol. Obs. Tonantzintla Tacubaya, 2, 16, 3 Karachentsev, I. D. 1972, A Catalogue of Isolated Pairs of Galaxies in the Northern Hemisphere, Astrofiz. Issled., 7, 3

Kazarian, M. A., \& Kazarian, E. S. 1980, Astrofizika, 16, 17

Kinman, T. D. 1984, in IAU Colloq. 78, Astronomy with Schmidt-Type Telescopes, ed. M. Capaccioli (Dordrecht: Reidel), 409

MacAlpine, G. M., Smith, S. B., \& Lewis, D. W. 1977, ApJS, 34, 95

Markarian, B. E. 1967, Astrofizika, 3, 55

Markarian, B. E., Lipovetskii, V. A., \& Stepanian, J. A. 1981, Astrofizika, 17,619

Markarian, B. E., Stepanian, J. A., \& Erastova, L. K. 1987, in IAU Symp. 121 , Observational Evidence of Activity in Galaxies, ed. E. Ye. Khachi-

kian, K. J. Fricke, \& J. Melnick (Dordrecht: Reidel), 25

Mazarella, J. M., \& Balzano, V. A. 1986, ApJS, 62, 751 


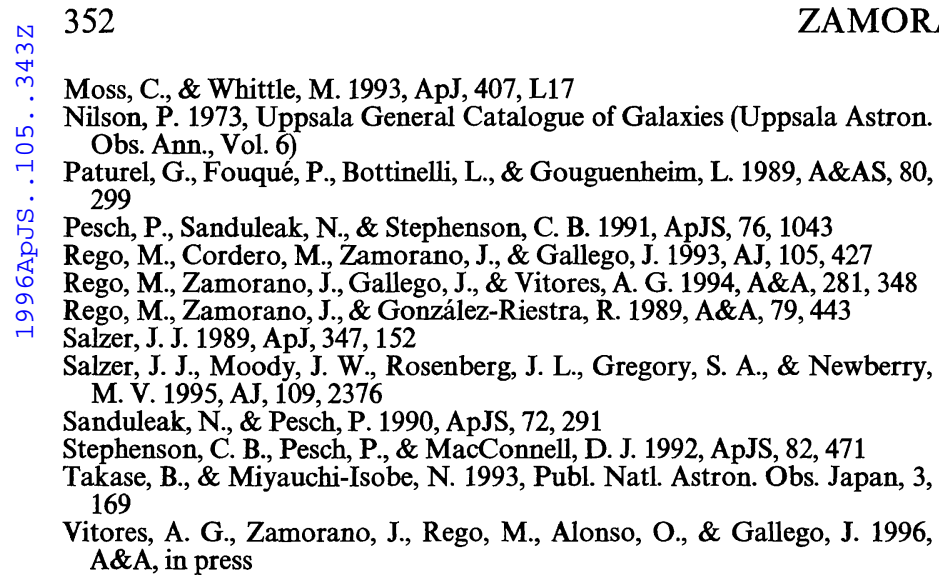
M. V. 1995, AJ, 109, 2376

Sanduleak, N., \& Pesch, P. 1990, ApJS, 72, 291

Stephenson, C. B., Pesch, P., \& MacConnell, D. J. 1992, ApJS, 82, 471

Takase, B., \& Miyauchi-Isobe, N. 1993, Publ. Natl. Astron. Obs. Japan, 3, 169

Vitores, A. G., Zamorano, J., Rego, M., Alonso, O., \& Gallego, J. 1996, A\&A, in press

Vorontsov-Velyaminov, B. A., Arkipova, V. P., \& Kranogorskaja, A. A. 1974, Morphological Catalogue of Galaxies, Vol. 5, (Moscow: Moscow State Univ.)

Wasilewski, A. J. 1983, ApJ, 272, 68

Weistrop, D., \& Downes, R. A. 1991, AJ, 102, 1680

Zamorano, J., Gallego, J., Rego, M., Vitores, A. G., \& González-Riestra, R. 1992, AJ, 104, 1000

Zamorano, J., Rego, M., Gallego, J., Vitores, A. G., González-Riestra, R., \& Rodríguez-Caderot, G. 1994, ApJS, 95, 387 (Paper I)

Zamorano, J., Rego, M., González-Riestra, R., Rodríguez-Caderot, G. 1990, Ap\&SS, 170, 353

Zwicky, F. 1971, Catalogue of Selected Compact Galaxies and of Posteruptive Galaxies (Gümligen: F. Zwicky)

Zwicky, F., Herzog, E., Wild, B., Karpowicz, M., \& Kowal, C. T. 19611968, Catalogue of Galaxies and of Clusters of Galaxies (Pasadena: Caltech) 